\title{
A simple estimation of glomerular filtration rate in children
}

\author{
Syarifuddin Rauf, MD; Husein Akbar, MD
}

\begin{abstract}
Background The estimation of glomerular filtration rate (GFR) is routinely used in the assessment of renal function in children. An accurate measurement of GFR is very helpful in detecting renal disease. The aim of this study was to compare GFR calculated by Schwartz's formula and that assessed by creatinine clearance measurement.

Methods A study to evaluate GFR calculated by the Schwartz's formula and by the conventional creatinine clearance method was done on $114(57 \%)$ boys and $86(43 \%)$ girls aged 6-13 years hospitalized in the Department of Child Health, Hasanuddin University, Wahidin Sudirohusodo Hospital, Makassar in 1996.

Results This study showed that there was no significant difference between the value of GFR determined by the conventional creatinine clearance method and that calculated by the Schwartz's formula according to age and sex. Our findings also did not show any difference of GFR assessed by both methods according to nutritional status of children. In regard to the accuracy of Schwartz's formula, the sensitivity, specificity, positive, and negative predictive values were $87.5 \%, 98.86 \%, 91.3 \%$, and $98.3 \%$, respectively. Conclusion It seems that Schwartz's formula might be used for assessing GFR in children particularly if either there is no facility for measuring creatininuria or there is difficulty in collecting complete and accurate-timing urine samples. The formula is a very simple and inexpensive method for determining GFR in children compared to the conventional creatinine clearance method [Paediatr Indones 2002;42:193-196].
\end{abstract}

Keywords: glomerular filtration rate, Schwartz's formula, creatinine clearance

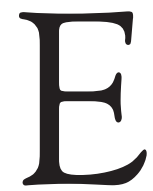

stimation of glomerular filtration rate (GFR) is routinely used in the assessment of renal function in children. The GFR is assessed based on the balance between glomerular hydrostatic and oncotic pressures, the permeability of the glomerular capillary and the surface area of the glomerulocapillary used for filtration. ${ }^{1,2}$ An accurate measurement of GFR is very helpful in detecting renal disease and in evaluating either the progress of the disease or the response to therapy. GFR in children can be assessed by the measurement of inulin, urea, EDTA or creatinine clearance, or by plasma concentration of urea and creatinine. ${ }^{3}$ The precise measurement of GFR is accomplished by quantifying the clearance of a substance that is freely filtered across the capillary wall and that is neither reabsorbed nor secreted by the tubules. The clearance (C) of such a substance is the volume of plasma that yields a quantity of the substance equal to that excreted in the urine over a specified time. Creatinine clearance (CCr) has been shown to be a valid measurement of GFR in children. ${ }^{4}$ However, the technique may become difficult because the need for collecting either complete or accurate-timing urine samples. Schwartz et al in 1976 reported that an accurate estimation of GFR could be obtained from the simple determination of plasma creatinine and body length. ${ }^{5}$

The purpose of our study was to compare GFR in children determined by the assessment of creatinine clearance to that calculated by the Schwartz's formula.

From the Department of Child Health, Medical School, Hasanuddin University- Wahidin Sudirohusodo Hospital, Makasar, South Sulawesi

Reprint requests to: Syarifuddin Rauf MD, Department of Child Health, Medical School, Hasanudin University; Wahidin Sudirohusodo Hospital, Makassar; Tel. 62-411-510676; Fax. 62-411-510088 


\section{Methods}

Subjects of this study were 200 children aged 6 to 13 years admitted to the Department of Child Health, Hasanuddin University/Wahidin Sudirohusodo Hospital, Makassar in 1996. The subjects' data including age and sex were recorded. Accurate 24hour urine samples were collected. Plasma creatinine $(\mathrm{PCr})$ and urine creatinine $(\mathrm{CrU})$ were measured by means of Jaffe's method. The GFR was determined based on two measurements. Firstly, it was assessed by conventional creatinine clearance method $(\mathrm{CCr})$ using the following formula:

\section{GFRCCr $=\underline{\operatorname{CrUxV}} \times \underline{1.73}\left(\mathrm{ml} / \mathrm{min} / 1.73 \mathrm{~m}^{2}\right)$ $\mathrm{PCr} \quad \mathrm{SA}$}

where the $\mathrm{CCr}$ denotes creatinine clearance, $\mathrm{CrU}$ reflects creatininuria (the amount of creatinine in a 24-hour urine sample), $\mathrm{V}$ represents the urinary flow rate $(\mathrm{ml} /$ minute), $\mathrm{PCr}$ equals to concentration of plasma creatinine, and SA stands for body surface area $\left(\mathrm{m}^{2}\right){ }^{6}$

Secondly, GFR was calculated by the Schwartz's formula as follows:

$$
\text { GFRSf }=0.55 \times \frac{\mathrm{H}(\mathrm{cm})}{\operatorname{PCr}(\mathrm{mg} / \mathrm{dl})} \quad\left(\mathrm{ml} / \mathrm{min} / 1.73 \mathrm{~m}^{2}\right)
$$

where $\mathrm{H}$ is for height and 0.55 is a constant of proportionality and equals $0.55 \mathrm{mg}$ creatinine per 100 $\mathrm{min} / \mathrm{cm} / 1.73 \mathrm{~m}^{2}$, for children aged 1 to 13 years. ${ }^{6,7}$ Chi square test and Student-t-test were used to compare GFR assessed by estimation of the $\mathrm{CCr}$ (GFRCCr) to that calculated by the Schwartz's (GFRSf). The $p$ value of $<0.05$ was considered as significant. Sensitivity, specificity, and negative and positive predictive values were used to assess the accuracy of the Schwartz's formula.

\section{Results}

During the period of 1996, 200 children aged 6 to 13 years were enrolled in this study, consisting of 114 boys (57\%) and 86 girls (43\%). One hundred and twenty one of them had good nutritional status $(60.5 \%)$; whereas $62(31 \%)$ and 17 (8.5\%) had moderate and poor nutritional status, respectively.

There was no significant difference between the mean GFR assessed by the conventional creatinine clearance and that calculated by the Schwartz's formula according to age and sex (Table 1). There was also no significant difference in GFR measured by both methods in children with good, moderate, or poor nutritional status.

Table 1. Relationship between GFRCCR and GFRSF

\begin{tabular}{lllll}
\hline Methods & N & Range & Mean & SD \\
\hline GFRCCr & 200 & $67.36-139.67$ & 92.91 & 13.43 \\
GFRSf & 200 & $68.59-139.33$ & 92.93 & 13.41 \\
\hline
\end{tabular}

$t=-0.13 ; d f=199 ; p=0.898$

Our study showed that 24 (12\%) out of 200 children evaluated by means of estimation of the CCr had GFR $<80 \mathrm{ml} / \mathrm{min} / 1.73 \mathrm{~m}^{2}$ and 176 (88 \%) had GFR $>80 \mathrm{ml} / \mathrm{min} / 1.73 \mathrm{~m}^{2}$. While $23(11.5 \%)$ out of 200 children evaluated by the Schwartz's formula had GFR $<80 \mathrm{ml} /$ menit $/ 1.73 \mathrm{~m}^{2}$ and the remaining had GFR $>80 \mathrm{ml} / \mathrm{min} / 1.73 \mathrm{~m}^{2}$.

Table 2. The accuracy of Schwartz's Formula (GFRSF) COMPARED TO THE GFRCCR AS THE GOLD STANDARD IN ASSESSING GFR IN CHILDREN

\begin{tabular}{llccc}
\hline & & \multicolumn{3}{c}{ GFR CCr } \\
\cline { 3 - 4 } & & decreased & normal & \\
\hline GFRSf & decreased & 21 & 2 & 23 \\
& normal & 3 & 174 & 177 \\
& Total & 24 & 176 & 200 \\
\hline
\end{tabular}

Normal = GFR $>80 \mathrm{ml} / \mathrm{min} / 1.73 \mathrm{~m}^{2} ;$ Decreased=GFR $<80 \mathrm{ml} / \mathrm{min} /$ $1.73 \mathrm{~m}^{2}$

The accuracy of GFR calculated by the Schwartz's formula compared to that assessed by the conventional creatinine clearance as the gold standard can be seen in Table 2 . The sensitivity, specificity, positive and negative predictive values of GFR calculated by the Schwartz's formula were $87.5 \%$, 98.9\%, 91.3\% and 98.3\%, respectively.

\section{Discussion}

The aim of our study was to compare GFR assessed by $\mathrm{CCr}$ to that calculated by Schwartz's formula derived from plasma creatinine and body length. Schwartz et 
al had done this investigation previously in children aged $1-13$ years using a constant $(\mathrm{k})$ of 0.55 . Counahan et al also conducted similar study but they used the constant of 0.43 . The plasma creatinine in the Schwartz's formula represented by a total value of creatinine but that in Counahan denoted the real value without non-creatinine chromogens. ${ }^{6-8}$ Zahcello et al investigated GFR in neonates estimated by $\mathrm{CCr}$ and compared with the values obtained by Schwartz's formula. ${ }^{9}$

In fact, the GFR is optimally measured by the clearance of inulin, but because of the technique is cumbersome, the GFR is commonly estimated by the clearance of endogenous creatinine. ${ }^{2,5}$ The creatinine clearance by far is the convenient method of obtaining a fairly accurate estimation of GFR. The clearance of pure creatinine, however, is slightly greater than that of inulin, indicating that some creatinine is actively secreted by the tubules. The amount of creatinine that is actively secreted is related to the level of plasma creatinine. The clearance of creatinine is much more convenient determination to make than that of inulin, as creatinin is already present in body fluids and its plasma concentration is remarkably steady throughout the 24 hours. Creatinine clearance test can therefore be performed over long periods without the necessity of continuous intravenous administration (note that inulin should be given intravenously), and because the plasma concentration is so constant that only one sample of blood needed to be taken for a 24-hour collection of urine. ${ }^{2,3} \mathrm{Al}$ though, the clearance of creatinine provides a good estimate of GFR in children, the use of this technique is limited by the need for obtaining complete and accurate-timing urine collections, especially in children. To overcome this problem, Schwartz et al derived a formula to estimate creatinine clearance in children aged 1 to 20 years from plasma creatinine concentration $(\mathrm{mg} / \mathrm{dl})$ and body length $(\mathrm{cm})$ without taking a 24-hour urine collection. ${ }^{4,6}$ The value of $\mathrm{k}$ depends on the relationship between muscle mass and growth as estimated from body weight. In full term infants $\mathrm{k}$ was found to be 0.45 because the ratio of muscle mass to body weight is lower than that in older children ${ }^{10}$.

Our results show that there was no significant difference between the value of GFR assessed by CCr and that calculated by the Schwartz's formula in accordance with age and sex (Table 1). The difference may be significant only in adolescence. It seems that body weight is greater and body length is higher in boys than in girls. The excretion of creatinine per kilogram body weight increases more rapidly in boys than in girls. Therefore, the constant $(\mathrm{k})$ increases in accordance with increasing age in boys and changes little in girls. For the same rate of GFR, adolescent boys had higher plasma creatinine concentrations and higher rates of creatinine excretion per kilogram body weight than girls and younger boys. ${ }^{6}$ Our study also did not find any difference of GFR assessed by CCr and that calculated by Schwartz's formula in accordance with nutritional status of children.

In regard to the accuracy of Schwartz's formula compared to conventional CCr as the gold standard, we found that the sensitivity, specificity, positive and negative predictive values were $88 \%, 99 \%, 91 \%$ and $98 \%$, respectively. In other words, if a child has a low GFR calculated by the Schwartz's formula, may be he or she has a decreased renal function. But if a child has a normal GFR determined by Schwartz's, there might be a little possibility that he or she has a decreased renal function. Seikaly et al (1996) concluded that Schwartz's formula seems to be capable only in estimating GFR in children with either normal renal function or mild renal insufficiency. ${ }^{11}$

Based on our results, we concluded that the Schwartz's formula might be used for assessing GFR in children, particularly if either there is no facility in measuring creatininuria or there is difficulty in collecting complete and accurate timing urine samples. Besides, the formula is a very simple and inexpensive method for determining GFR in children.

\section{References}

1. Cony HE, Spitzer A. Renal blood flow and glomerular filtration rate during development. ln: Edelman C Jr, editor. Pediatric kidney disease. Boston: Little Brown; 1994. p. 49-59.

2. Davies JG, Taylor CM, White RHR, Marshall T. Clinical limitations of the estimation of glomerular filtration rate from height/plasma creatinine ratio: a comparison with simultanoeus $51 \mathrm{Cr}$ edetic acid slope clearance. Arch Dis Childh 1982;57:607-10. 


\section{Paediatrica Indonesiana}

3. Behrman RE, Kliegman RM. The urinary system and pediatric gynecology. In: Behrman RE, Kliegman RM, editors. Nelson textbook of pediatrics. 14th ed. Philadelphia: Saunders; 1992. p. 1323-5

4. Goldsmith Dl. Clinical and laboratory evaluation of renal function. In: Edelman CM, Jr, editor. Pediatric kidney disease. Boston: Little Brown; 1978. p. 213-24

5. Schwartz GJ, Haycock GB, Edelman CM, Spitzer A. A simple estimate of GFR in children derived from body length and plasma creatinine. Pediatrics 1976;58:259.

6. Schwartz GJ, Gauthier B. A simple estimate of glomerular filtration rate in adolescent boys. J Pediatr 1985;106:522-6.
7. Counahan R, Chantler C, Ghazali S, Kirkwood B, Rose F, Barratt TM. Estimation of glomerular filtration rate from plasma creatinine concentration in children. Arch Dis Childh 1976;51:875-8.

8. Barret LM, Chantler C. Clinical assessment of renal function. In: Rubin MI, Barret TM, editors. Pediatric nephrology. Baltimore: Williams \& Wilkins; 1975. p. 55-63.

9. Schwarzt GJ, Feld LG, Lang ford DJ. A simple estimate of glomerular filtration rate in full term infants during the first year of life. J Pediatr 1984;6:849-54.

10. Seikaly MG, Browne R, Bajaj G, Arant BS Jr. Limitations to body length/serum creatinine ratio as an estimate of glomerular filtration in children. Pediatr Nephrol 1996;10:709-11 